Article

\title{
De Novo Transcriptome Sequencing of Oryza officinalis Wall ex Watt to Identify Disease-Resistance Genes
}

\author{
Bin $\mathrm{He}^{1}$, Yinghong $\mathrm{Gu}^{1}$, Xiang Tao ${ }^{2}$, Xiaojie Cheng ${ }^{1}$, Changhe Wei ${ }^{1}$, Jian Fu ${ }^{3}$, \\ Zaiquan Cheng ${ }^{3, *}$ and Yizheng Zhang ${ }^{1, *}$
}

Received: 1 November 2015; Accepted: 3 December 2015; Published: 10 December 2015

Academic Editor: Marcello Iriti

1 Key Laboratory of Bio-Resources and Eco-Environment, Ministry of Education, Sichuan Key Laboratory of Molecular Biology and Biotechnology, College of Life Sciences, Sichuan University, Chengdu 610064, China; hebin.li@foxmail.com (B.H.); yinghonggu@gmail.com (Y.G.); xiaoxiaojie1991@foxmail.com (X.C.); hantanheying@126.com (C.W.)

2 Chengdu Institute of Biology, Chinese Academy of Sciences, Chengdu 610041, China; taoxiang180@gmail.com

3 Biotechnology \& Genetic Resources Institute, Yunnan Academy of Agricultural Sciences, Kunming 650223, China; fugein@aliyun.com

* Correspondence: czquan-99@163.com (Z.C.); yizzhang@scu.edu.cn (Y.Z.); Tel.: +86-871-514-0200 (Z.C. \& Y.Z.)

\begin{abstract}
Oryza officinalis Wall ex Watt is one of the most important wild relatives of cultivated rice and exhibits high resistance to many diseases. It has been used as a source of genes for introgression into cultivated rice. However, there are limited genomic resources and little genetic information publicly reported for this species. To better understand the pathways and factors involved in disease resistance and accelerating the process of rice breeding, we carried out a de novo transcriptome sequencing of $O$. officinalis. In this research, 137,229 contigs were obtained ranging from 200 to 19,214 bp with an N50 of 2331 bp through de novo assembly of leaves, stems and roots in O. officinalis using an Illumina HiSeq 2000 platform. Based on sequence similarity searches against a non-redundant protein database, a total of 88,249 contigs were annotated with gene descriptions and 75,589 transcripts were further assigned to GO terms. Candidate genes for plant-pathogen interaction and plant hormones regulation pathways involved in disease-resistance were identified. Further analyses of gene expression profiles showed that the majority of genes related to disease resistance were all expressed in the three tissues. In addition, there are two kinds of rice bacterial blight-resistant genes in O. officinalis, including two Xa1 genes and three Xa26 genes. All 2 Xa1 genes showed the highest expression level in stem, whereas one of Xa26 was expressed dominantly in leaf and other 2 Xa26 genes displayed low expression level in all three tissues. This transcriptomic database provides an opportunity for identifying the genes involved in disease-resistance and will provide a basis for studying functional genomics of $O$. officinalis and genetic improvement of cultivated rice in the future.
\end{abstract}

Keywords: Oryza officinalis; transcriptome; de novo assembly; disease-resistant genes

\section{Introduction}

Oryza officinalis Wall ex Watt (CC, $2 \mathrm{n}=2 \mathrm{x}=24)$ is one of the important wild species in Oryza genus. It is one of the three wild species indigenous in China [1]. It has reservoirs of many useful genes for rice breeding, such as resistance to blast, bacterial blight (BB), brown planthopper (BPH), and white backed planthopper (WBPH) [2-5]. It can also tolerate drought, cold, and other abiotic stresses [6,7]. 
Through interspecific hybridization and backcrossing between O. officinalis and Oryza sativa, a number of resistance genes have been introduced into cultivars and some varieties have been released for commercial cultivation. In 2007, several introgression lines from an O. sativa $\times$ O. officinalis cross were produced to enhance blast resistance ability by Jena and Khush [8]. One of the most successful examples of the transfer of genes from $O$. officinalis is that of the resistance genes to brown planthopper (BPH). Four BPH resistance genes, Bph10, Bph18, bph11, and bph12, were transferred from O. officinalis to cultivated rice. Four breeding lines were released as varieties (MTL95, MTL98, MTL103, and MTL110) for commercial cultivation in Mekong Delta, Vietnam [9]. In addition, Huang et al. also transferred the $\mathrm{BPH}$ resistance gene from O. officinalis into Zhensheng $97 \mathrm{~B}$ to improve disease resistance [10]. However, the present understanding of the disease resistance mechanism and related disease-resistance genes in O. officinalis is limited.

Successful implementation of a defense response requires that plants respond to a pathogen rapidly and accurately. Signaling events in the plant-pathogen interaction are critical for the process of pathogen recognition and lead to hypersensitive response (HR). During the process, a first layer is based on the amazingly sensitive perception of pathogen/microbe-associated molecular patterns (PAMPs or MAMPs) through pattern recognition receptors (PRRs) at the plant's cell surface. The second layer of defense is called effector-triggered immunity (ETI), in which plants, in turn, can recognize such effectors through additional receptors, particularly nucleotide-binding leucine-rich repeat (NB-LRR) proteins [11]. Besides, evidence has accumulated over the past few years to indicate that plant hormones, such as salicylic acid (SA), jasmonic acid (JA) and abscisic acid (ABA), also play an important regulatory role in the plant immune response, such as determining the recognition of PAMPs, mediating system-acquired resistance and activating the hypersensitive response. Related genes on the plant-pathogen interaction and the plant hormones regulation were reported in some organisms [12].

More recently, genome-wide approaches have become valuable tools in characterizing gene expression and elucidating the genetic networks of disease resistance at a global level [13]. In the past, the draft genome sequence of the O. sativa ssp. indica cv. 9311 and the full genome sequence of $O$. sativa ssp. japonica cv. Nipponbare were completed through a whole-genome shotgun sequencing approach and a map-based sequencing strategy, respectively [14,15]. Even so, the cost of genome sequencing is too high to afford. Therefore, much effort was involved in rice EST projects. Approximately 1,249,110 ESTs and more than 50,000 FLcDNA sequences of cultivated rice are currently available in public databases. However, the genomic studies of rice wild relatives are still in their infancy with the exception of the generation of 1888 leaf FLcDNAs from the O. rufipogon (AA genome), 5211 leaf ESTs from the O. minuta (BBCC genome), 68,132 leaf unigenes from O. officinalis (CC genome) and 71,367 root unique sequences from the O. longistaminata (AA genome) [7,16-18]. Furthermore, genes that might be expressed in different organs are underrepresented in EST studies.

To further explore the O. officinalis transcriptome, we employed RNA-seq for three tissues, i.e., leaves, stems and roots. In this study, we provide a more comprehensive transcriptome of $O$. officinalis using the next-generation sequencing platform Illumina. The large EST dataset will remarkably enlarge genomic resources of $O$. officinalis available in the public database. The transcriptome data have led to the identification of a number of candidate genes and functional elements determining plant-pathogen interaction and plant hormone regulation pathways associated with diseases resistance, which are of great importance to efforts for genetic improvement of the cultivated rice.

\section{Results and Discussion}

\subsection{De Novo Assembly and Quality Assessment}

To develop an overview of the $O$. officinalis transcriptome and to obtain the representative transcripts, three tissues representing various vegetative tissues, including leaves, stems and roots, 
were harvested for RNA isolation. Following the Illumina manufacturer's instructions, the shotgun libraries were constructed and used for sequencing with the Illumina High-Seq 2000 platform. A total of 54,941,770, 54,282,656 and 53,625,698 raw reads with a length of 90 bp were generated from $O$. officinalis leaves, stems and roots transcripts, respectively. After quality checks and trimming of the adapter, the assembly strategy of single-assembler multiple-parameters (SAMP) from Oases was employed to assemble the transcriptome on the basis of our previous study [19]. Finally, a total of 137,229 contigs were generated ranging from 200 to 19,214 bp with an N50 of $2331 \mathrm{bp}$ (Table 1). The majority of the assemblies were over $500 \mathrm{bp}$ in size (71\%) and $28 \%$ of them were longer than $2000 \mathrm{bp}$.

Table 1. Summary of sequence assembly and function annotation of the O. officinalis transcriptome.

\begin{tabular}{ll}
\hline Items & Number of Sequences \\
\hline Assembly & \\
\hline Total number of contigs & 137,299 \\
Mean length (bp) & 1459 \\
Maximum contig length $(\mathrm{bp})$ & 19,214 \\
N50 (bp) & 2331 \\
Number of contigs $(\geqslant 1 \mathrm{~kb})$ & 72,521 \\
\hline Annotation & \\
\hline Number of predicted ORFs & 82,983 \\
Number of predicted ORFs $(\geqslant 900 \mathrm{bp})$ & 53,239 \\
Transcript BLASTx against NR & 88,249 \\
Transcript BLASTx against Pfam & 79,310 \\
Transcripts annotated with GO terms & 75,589 \\
Transcript annotations against KEGG & 21,191 \\
\hline
\end{tabular}

To assess how well the assembled sequences represent the actual transcriptome population, several approaches have been adopted. First, the transcriptome gene coverage was judged by comparison with the sequence information available for $O$. officinalis and phylogenetically related species, O. sativa. 58 of 69 complete protein-coding genes from O. officinalis and 429 of 523 proteins from $O$. sativa in the NCBI database were present in our assembled transcriptome. We then detected the transcript sequence quality and completeness through ORF prediction, since the optimal assembly results could produce long and complete ORFs, and as many as possible. A high proportion of the transcripts $(82,983,61 \%)$ could be detected in ORFs in which 53,239 $(64 \%)$ ORFs are longer than $900 \mathrm{bp}$. Thirdly, all reads were mapped to the contigs for counting the utilization ratio. $92.52 \%$ of them were found to be aligned back to the contigs, indicating that almost all reads were utilized for the de novo assembly. In addition, the accuracy of the assembled contigs was up to $82 \%$. On the basis of the above evaluation, the quality of the assembled transcriptome is good enough for functional annotation and further analysis.

\subsection{Functional Annotation of O. officinalis Transcriptome}

To predict and analyze the function of the transcripts of $O$. officinalis, all contig sequences were first aligned against the NCBI non-redundant (NR) protein database using BLASTx. A total of 88,249 significant BLAST top hits were returned with a cut-off $E$-value of $1 \times 10^{-5}(64 \%$ of all transcripts; Table 1). As shown in the previous study, the length of contigs is crucial in determining the efficiency of BLAST searches [20]. Our results showed that $99 \%$ of the matching efficiency were observed for sequences longer than $2000 \mathrm{bp}$ and $96 \%$ for those between 1500 to $2000 \mathrm{bp}$, whereas the matching efficiency decreased to about $71 \%$ for those ranging from 500 to $1000 \mathrm{bp}$ and to $23 \%$ for sequences between 200 to $500 \mathrm{bp}$ (Figure 1a). For species distribution, most of the sequences $(85 \%)$ had the top matches with those from the Oryza sativa, followed by the Zea mays $(1.5 \%)$, Brachypodium distachyon (1.2\%), Sorghum bicolor (1.2\%) and Hordeum vulgare (0.5\%) (Figure 1b).

To functionally classify the O. officinalis transcripts, sequences that had matches in NR databases achieved GO annotations with the Uniprot database. Of the 88,249 transcripts with NR annotation, 
a total of 75,589 transcripts were assigned to 369,110 GO terms. The distribution of GO terms for molecular functions, biological processes and cellular components is shown in Figure 2. Except the general categories, for the biological process classification, genes involved in the "biological regulation" (GO: 0065007) and "pigmentation" (GO: 0043473) were the most highly represented categories. For the molecular function classification, "binding functions" (GO: 0005488) was the most enriched GO term, followed by "catalytic functions" (GO: 0003824). For the cellular component, the major categories were "macromolecular complex" (GO: 0032991), "envelope" (GO: 0031975) and "organelle" (GO: 0043226).
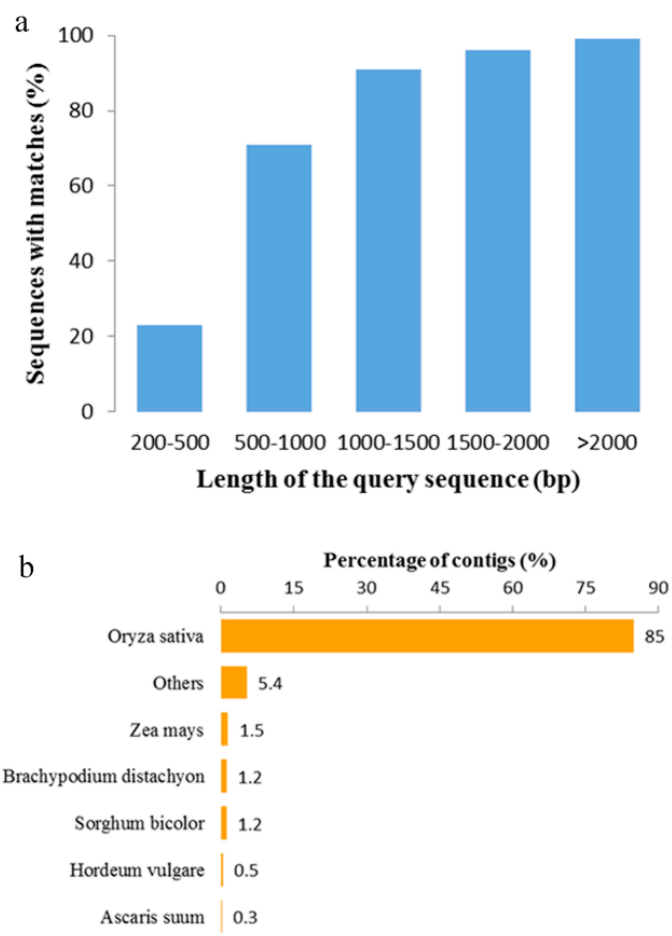

Figure 1. Characteristics of the homology search of contigs against the NR database. (a) Effects of query sequence length on percentage of significant matches. The cut-off value was set at $1.0 \times 10^{-5}$. The proportion of sequences with matches in the NR database at NCBI is greater among the longer assembled sequences; (b) Species distribution is shown as the percentage of the total homologous sequences.

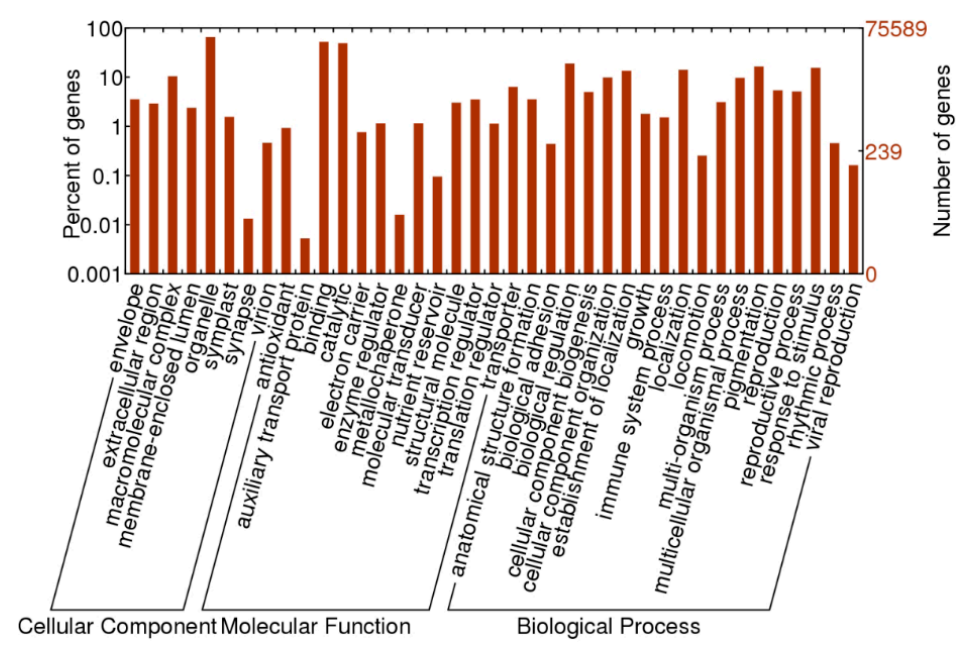

Figure 2. Gene Ontology classification of all O. officinalis transcripts. 


\subsection{Domain Prediction and KEGG Pathway Mapping}

Considering that the information of conserved domains within a gene was indicative of deducing genes' function, we performed the annotation of potential domains inside the assembled transcripts. To facilitate this procedure, ORFs for each transcript were extracted using a set of programs included in the Trinity package (see Methods), and then all the transcripts with predicted ORFs were searched against the PFAM database using HMMER v3.0 locally. Overall, a total of 79,310 transcripts were categorized into 6384 domains/families. Based on the frequency of the occurrence of transcripts contained in each Pfam domain, the top thirteen abundant domains/families were ranked and listed in Figure 3a. Among these domains/families, the "protein kinasedomain" and its subclass "protein tyrosine kinase" play an important role in a multitude of cellular processes, including division, proliferation, apoptosis, and differentiation; proteins with the "leucine rich repeats" domain are recognized to be frequently involved in signaling, translation and protein-binding; and the "NB-ARC domain" has been reported to be shared by plant resistance gene products. Other protein families, such as "RNA recognition motif", "WD domain, DEAD/DEAH box helicase, TPR repeat", "cytochrome P450" and so on, which have some basic functions in plants, were also found in the top thirteen items on the list.

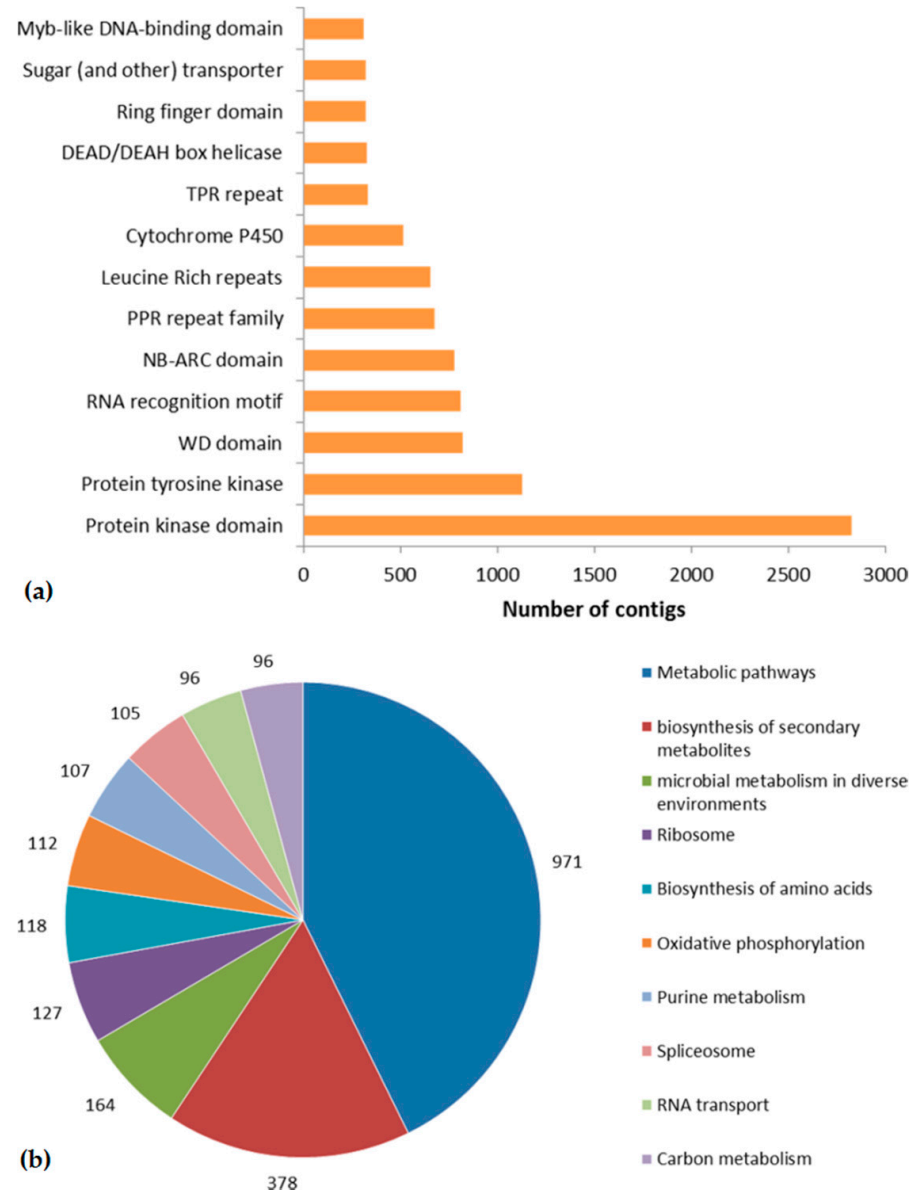

Figure 3. Domains prediction and KEGG classification of assembled contigs. (a) The 13 most abundant protein families in O. officinalis; (b) The top 10 pathways with the highest contig numbers.

To fully identify the biological pathways that are active in O. officinalis, all the assembled sequences were assigned with Kyoto Encyclopedia of Genes and Genomes orthology (KO) identifiers using a KEGG Automatic Annotation Server (KAAS) with the single-directional best hit information method, and subsequently mapped to pathways and enzymes using the KEGG API. As a result, 
a total of 21,191 assembled sequences were found to be involved in 341 predicted KEGG metabolic pathways. The pathways with the most representation by the unique sequences were metabolic pathways (971 members), biosynthesis of secondary metabolites (378) and microbial metabolism in diverse environments (164). Figure $3 \mathrm{~b}$ summarized the top 10 pathways with the most contigs. These results provide a valuable resource for investigating metabolic pathways in O. officinalis.

\subsection{Genes Related to Disease Resistance}

O. officinalis has been reported as a source of resistance to blast, bacterial blight, white-backed and brown planthoppers [4,5]. Therefore, it is very important to know the genes involved in plant resistance to disease in O. officinalis with regard to the following aspects: (1) the signaling events in the plant-pathogen interaction and their involvement in pathogen recognition; (2) the central role of plant hormones in the regulation of defense responses to pathogen; (3) and the specific examples of resistance genes, such as genes related to bacterial blight resistance.

\subsubsection{Signaling Events in the Plant-Pathogen/Insect Interaction}

Although the plant-pathogen interaction pathway has been known in O. sativa, existing knowledge on the pathways and enzymes involved in plant-pathogen interaction in wild rice, such as O. officinalis, is still limited. Figure 4 shows the plant-pathogen interaction pathway reconstructed based on the determined transcripts, and Table S1 lists all the transcripts.

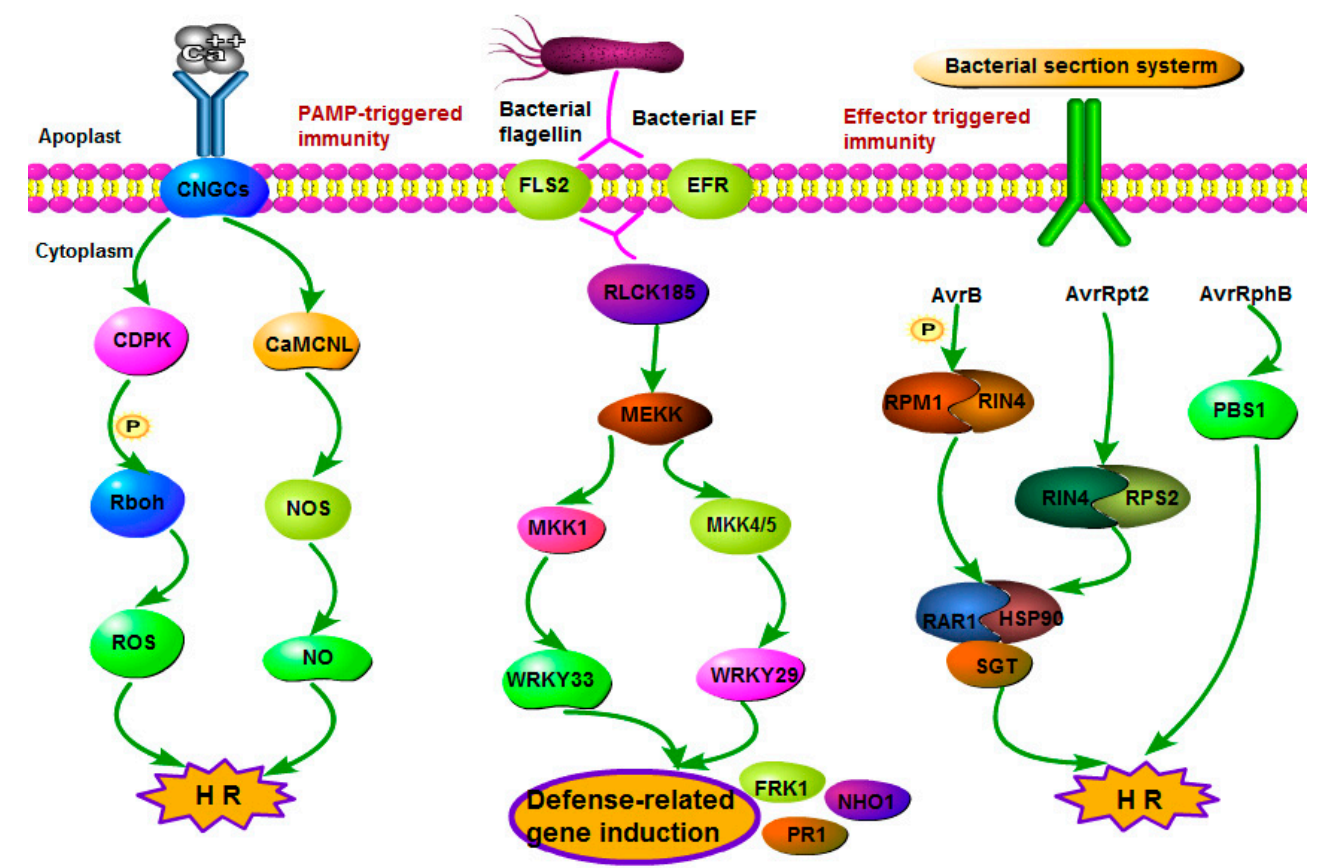

Figure 4. The plant-pathogen interaction pathway predicted in O. officinalis. This pathway is divided into two sub-pathway: PTI and ETI. The genes and transcription factors listed in this figure are all identified in O. officinalis.

All plants can recognize pathogen-associated molecular patterns (PAMPs) with multiple layers against invading pathogens. The primary response includes the perception of pathogens by cell-surface pattern-recognition receptors (PRRs) and is referred to as PAMP-triggered immunity (PTI). In addition to PRRs that recognize PAMPs, plants can also rely on immune receptors that recognize effectors or host plant targets manipulated by effectors resulting in effector-triggered immunity (ETI) [21]. Besides, the level of $\mathrm{Ca}^{2+}$ concentration is also a regulator for production of reactive oxygen species, cell wall reinforcement and hypersensitive response [22]. Based on the 
transcriptome annotation, in this study, we identified all of the genes encoding enzymes and proteins involved in the plant-pathogen interaction pathway in both O. sativa and O. officinalis. Moreover, three additional proteins are involved in the transcriptome of $O$. officinalis: WRKY transcription factor 29 (WRKY29: Contig_52053) and two R proteins (RAR: Contig_66753; FPK: Contig_15502), which may present novel genes expressed especially in O. officinalis but not in O. sativa. Recent studies revealed that OsPUB44 (Os06g10660), a rice ubiquitin E3 ligase, and OsRLCK185 (Os09g27890), a rice receptor-like cytoplasmic kinase, positively regulate immune responses [23]. In O. officinalis, we also identified one gene (Contig_47198) homologous to OsPUB44 with identity of $98 \%$ and two genes (Contig_23654 and Contig_26247) homologous to OsRLCK185 with identity of $89 \%$ and $84 \%$. In addition, there is evidence that OsRLCK185 transmits the immune signal from OsCERK1 to the downstream mitogen-activated protein kinase (MAPK) cascade, while OsPUB44 functions in a different pathway than OsRLCK185 or downstream of the MAPK cascade [24]. Furthermore, some research has reported that LYP4 (Os09g27890) and LYP6 (Os06g10660) play a role in peptidoglycan perception and resistance against Xoo (Xanthomonas oryzae pv. oryzae) [25]. As expected, we identified one gene (Contig_60426) homologous to LYP4 with identity of 95\% and one gene (Contig_59080) homologous to LYP6 with identity of $97 \%$ in O. officinalis. Although the previous research reported that transcription of these two genes could be induced rapidly upon exposure to bacterial pathogens or diverse MAMPs, their specific locations in PTI were not fully understood.

The results from expression analysis of genes related to plant-pathogen interaction in different tissues showed that CNGCs and RIN4 had a very low expression level in the three tissues. In addition, there were two genes, NOS and RPM1, only expressed in leaves. The other genes related to plant-pathogen interaction were all expressed in the three tissues (Table S1).

\subsubsection{Regulation of Defense Responses by Plant Hormones}

To cope with destructive diseases, plants have evolved molecular mechanisms mediated by multiple, highly regulated and coordinated signaling networks that rely on plant hormones, including jasmonic acid (JA), salicylic acid (SA) and abscisic acid (ABA), as secondary messengers.

Reviews of SA biosynthesis and signal transduction mechanisms has discussed its important regulatory role in mediating system-acquired resistance, activating the hypersensitive response and recognition of PAMPs, as well as activation of ETI [26]. In addition, when inflicted by chewing insects or mechanical damage, JA would be accumulated rapidly $(<30 \mathrm{~min})$ at the site of wounding [27]. In O. sativa, the routes of JA metabolism that modulate plant responses to disease are as follows: (1) Once JA have been synthesized through linolenic acid metabolism, it formed jasmonoyl-isoleucine (JA-Ile) and other JA-amino acid conjugates (JACs) through jasmonate resistance (JAR) and related conjugating enzymes; (2) the signal transduction of amino acid-conjugated forms of JA resulted in the formation of a coronatine-insensitive1 (COI1)-JA-JAZ ternary complex, a crucial node in downstream signaling pathways of JA, in which JAZs are then ubiquitinated and degraded; (3) rapid resynthesis of JAZ repressors presumably provides a mechanism to the activation of transcriptional regulons and potentially cell-damaging defensive processes. In the annotated transcriptome of O. officinalis, all of the key enzymes and proteins involved in the JA signal transduction were identified (Figure 5 and Table S2).

On the other hand, salicylic acid is also a key regulator of plant defense responses. In plants, the application of SA or SA analogs results in pathogenesis-related (PR) gene expression and then enhances disease resistance [28,29]. Firstly, accumulation of SA from phenylalanine metabolism induces a change in cellular redox potential triggering the reduction of non-expressed pathogenesis-related1 (NPR1) to active monomers which are then translocated to the nucleus and interacted with TGA transcription factors. Then, the binding of TGA factors to SA-responsive elements in the promoters of PR genes is stimulated by these interactions, and the subsequent transcriptional reprogramming leads to the activation of systemic acquired resistance (SAR). As proved in rice, the overexpression in rice of NPR1 confers enhanced resistance 
to Xanthomonas Oryzae pv. Oryzae (Xoo). In addition, recent studies revealed that OsWRKY45 plays a crucial role in NPR1-independent SA signaling in cultivated rice, and two allelic genes, OsWRKY45-1 (from japonica) and OsWRKY45-2 (from indica), which encode proteins with a 10-amino acid difference, play opposite roles in resistance against Xoo [30,31]. Here we identified one gene (Contig_52040) homologous to OsWRKY45. When comparing with the two allelic genes, OsWRKY45-1 (GenBank: GQ331932) and OsWRKY45-2 (GQ331927), OsWRKY45 in O. officinalis showed a difference with OsWRKY45-1 (from japonica) and OsWRKY45-2 (from indica) (Figure S1). Therefore, we inferred that OsWRKY45 may express in another allelic form neither OsWRKY45-1 nor OsWRKY45-2 in O. officinalis. Furthermore, the NPR1, TGA and PR1 from the transcriptome of O. officinalis involved in the SA signal transduction pathway were identified (Figure 5 and Table S2).

Abscisic acid (ABA) is a critical signaling molecule to activate disease resistance in different plant species through regulating various aspects of plant growth and development [32]. Hence, the biological and agricultural importance of ABA has led to increasing attention regarding its mechanism of signal transduction, and many putative signal transducers have been reported [33]. The PYR/PYL family proteins inhibit protein phosphatase 2C (PP2C) in an ABA-dependent manner as ABA receptors. Subsequently, PP2C interacts with a protein kinase, SnRK2, which is activated by osmotic stress or $\mathrm{ABA}$ and positively regulates the $\mathrm{ABA}$ response in various tissues. Furthermore, this complex stimulates the expression of $\mathrm{ABA}$ responsive element binding factor $(\mathrm{ABF})$, which encodes a basic leucine zipper transcription factor. Finally, the expression of ABF enhanced resistance to necrotrophic pathogens. There is overwhelming evidence that high ABA concentrations interfere with resistance against pathogens controlled by the SA signaling pathway. Likewise, the genes related to SA signaling pathway were all detected in our O. officinalis transcriptome (Figure 5 and Table S2).

To assess the expression level of genes that are related to the regulation of defense responses by plant hormones (four for JA regulation, three for SA regulation and four for ABA regulation), differential expression analysis in three tissues was performed. From the results, JAR showed a very low expression level in the three tissues, while NPR1 displayed a high expression level. In addition, the differential expression analysis revealed that almost all of these genes related to the regulation of defense responses by plant hormones displayed a high expression level in three tissues, except JAZ, which was not expressed in leaves, and SnRK2, which did not exist in stems (Table S2). The results may reveal that plant hormones play important roles in defense responses and their regulation was not restricted to one tissue.

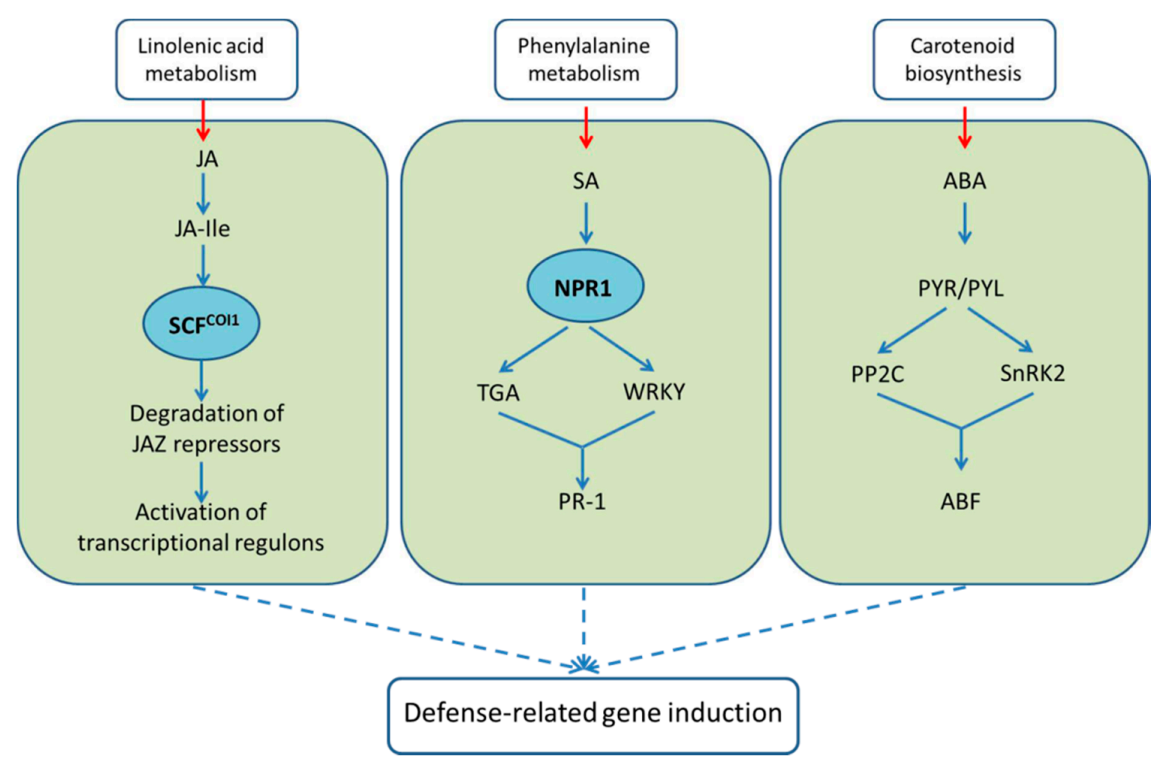

Figure 5. Genes of $O$. officinalis involved in the three plant hormones regulation pathways. The genes and transcription factors listed in this figure were all identified in O. officinalis. 


\subsubsection{Genes Related to Bacterial Blight Resistance}

Bacterial blight, caused by Xanthomonas Oryzae pv. Oryzae (Xoo), is a devastating disease affecting rice worldwide. So far, seven bacterial blight resistance genes have been cloned and sequenced (Xa1, xa5, xa13, Xa21, Xa23, Xa26 and Xa27) [34]. It is known that wild rice O. officinalis is resistant to bacterial blight. Therefore, we also identified the contigs involved in the bacterial blight resistance from $O$. officinalis transcripts. However, there were only 2 types of bacterial blight-resistance genes (Xa1 and $\mathrm{Xa26}$ ) identified in O. officinalis transcriptome. Two contigs (Contig 13873, Contig 1227) were annotated as bacterial blight-resistance protein Xa1 (named OofXa1_a,b), while three contigs (Contig 67329, Contig 77980, Contig 79023) were annotated as bacterial blight-resistance protein Xa26 (named OofXa26_a,b,c). However, the sequence of OofXa26_a,b,c were not complete. The results from expression analysis in different tissues showed that both Xa1 genes had the highest expression level in stem, whereas OofXa26_a was expressed dominantly in leaf and the other two Xa26 genes displayed low expression levels in all the three tissues.

The phylogenetic relationship of Xa1 in O. officinalis transcriptome was also investigated. It was clearly shown that the phylogenetic tree is divided into two groups. Likewise, OofXa1_a and OofXa1_b were divided into two groups, in which OofXa1_a was clustered into the branch of Oryza sativa Japonica Group and OofXa1_b was clustered into the other branch (Figure 6).

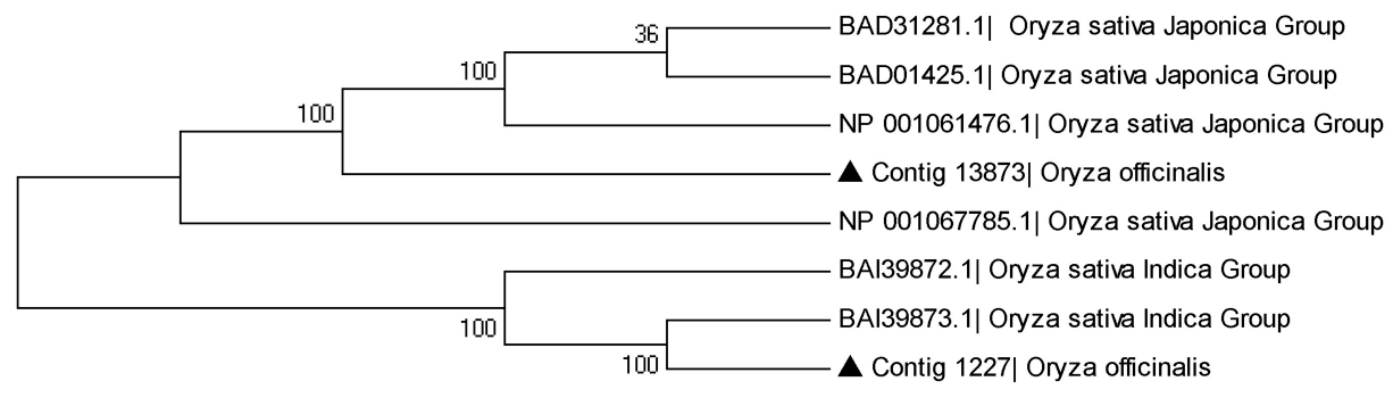

Figure 6. Phylogenetic relationship of the bacterial blight resistance protein Xa1 of O. officinalis with those of other species of the Oryza genus. Triangle symbol: OofXa1.

\section{Materials and Methods}

\subsection{Plant Materials and RNA Extraction}

O. officinalis was planted at natural temperature and light in Yuanjiang, Yunnan Province of China. Three different tissues including mature leaves, stems and roots of O. officinalis, which were kindly provided by Zaiquan Cheng (Yunnan Academy of Agricultural Sciences, Kunming, China), were harvested when flowering. At least three independent biological replicates of each tissue sample were collected, snap-frozen immediately and then stored at $-70{ }^{\circ} \mathrm{C}$ until processing. Total RNA was extracted with TRIzol Reagent (Invitrogen, Carlsbad, CA, USA) following the manufacturer's instructions and treated with DNase I (Fermentas, Burlington, ON, Canada). After further extraction to remove the protein of DNase I, RNA integrity was confirmed using the Agilent 2100 Bioanalyzer (Agilent, Santa Clara, CA, USA) with a minimum integrity number value of 8 .

\section{2. cDNA Library Construction and Sequencing}

The poly (A) + RNA (mRNA) was isolated and purified using Dynal oligo(dT) 25 magnetic beads (Invitrogen) following the manufacturer's instructions. Followed by purification, the mRNA was fragmented into smaller pieces by the fragmentation buffer. Then the first-strand cDNA was synthesized using SuperScript III reverse transcriptase and N6 random hexamers based on the cleaved RNA fragments. Subsequently, second strand cDNA synthesis was performed by dNTPs, 
DNA Polymerase I and RNase H. The products were purified using AMPure XP beads and further processed by the ligation of adapters and an end repair. The purified products were enriched with PCR for preparing the final sequencing library. The quality control analysis of the cDNA library was performed by Agilent 2100 Bioanalyzer. The cDNA library was sequenced from both $5^{\prime}$ and 3' ends using the Illumina HiSeq 2000 platform (Illumina, Dedham, MA, USA) according to the manufacturer's protocol. Then the Illumina sequencing by-synthesis, image analysis and base-calling procedures were used to obtain paired-end (PE) read sequence information and base-calling quality values, in which $90 \mathrm{bp}$ PE reads were obtained [35].

\subsection{Data-Preprocessing, De Novo Assembly and Assembly Assessment}

The raw reads were first filtered to remove adaptors, poly-A tails and contaminants using the FASTX tool kit (v 0.0.13, Gordon, LongIsland, NY, USA). Short (length $<25$ bp) reads and Low-quality (phred score $<20$ ) were trimmed by SolexaQA package $(-125 ;-\mathrm{h} 20)$ [36]. FASTQC (v 0.10.1, Andrews, Edinburgh, UK) was further used to detect the quality of the retained pair-end reads [37]. Clean reads were then de novo assembled using Single-Assembler Multiple-Parameters of Oases, which is an optimal assembly strategy for diploid organisms [19]. In order to evaluate the quality of assembly, four criterion were used: (i) gene coverage; (ii) transcript sequence quality and completeness; (iii) utilization ratio of reads; (iv) accuracy.

The gene coverage was judged by comparison with the sequence information available for O. officinalis and $O$. sativa, phylogenetically related species. There were 69 complete protein-coding genes from $O$. officinalis were all selected and 523 proteins from $O$. sativa in the NCBI database were randomly chosen as reference databases. The megablast and the common Perl analysis script were used to analyze the representation. Transcriptome quality was assessed by the best candidate coding sequence (CDS) for each contig using the TransDecoder program, which is included in the Trinity package [38]. To evaluate the utilization ratio of reads, all of the clean PE reads were aligned back to these contigs by using Bowtie2 (v2.0.0-beta5) program [39]. Finally, the accuracy was calculated with $\mathrm{Acc}=\mathrm{TP} /(\mathrm{TP}+\mathrm{FN})(\mathrm{TP}=$ true positives, $\mathrm{FN}=$ false negatives $)$, where Acc is accuracy, $\mathrm{FN}$ is the sum of all reference segment length that were not aligned, and TP is the sum of all aligned segment length (the overlap aligned regions were only calculated once) [40].

\subsection{Gene Annotation and Differential Gene Expression Analysis}

The assembled contigs were annotated by aligning against the NR databases using the BLASTX algorithm with a significance threshold of $E$-value of $10^{-5}$, retrieving proteins with the highest sequence similarity. To further annotate transcripts, the best Blastx hit from NR database for each transcript was submitted to the BLAST2GO platform to obtain GO terms [41]. WEGO (Web Gene Ontology Annotation Plot) is a useful tool for plotting GO annotation results and has been widely used in many important biological research projects. Therefore, we achieved GO classification through submitting GO annotation files in WEGO native format to WEGO [42]. Kyoto Encyclopedia of Genes and Genomes (KEGG) pathway mapping is the process to map molecular datasets, especially large-scale datasets in genomics, transcriptomics, proteomics, and metabolomics, to the KEGG pathway maps for biological interpretaion of higher-level systemic functions [43]. The assembled sequences were submitted to the online KEGG Automatic Annotation Server (KAAS) with single-directional best hit (SBH) method [44]. The output includes KO assignments and KEGG pathways that are involved with the KO assignments. For Pfam domain annotation, the predicted protein sequences were searched against the Pfam-A database (v27.0, Bateman, Hinxton, UK) using HMMER v3.0 (Johnson, MO, USA) locally [45].

Transcript abundances in O. officinalis leaf, stem and root tissues were estimated using edgeR in fragments per kilobase of transcript per million (FPKM) units. Genes that were differentially expressed among leaf, stem and root tissues were considered to be significantly different at an FDR 
(false discovery rate) $<0.001$, and an absolute value of $\log _{2}$ fold-change $\left(\log _{2} \mathrm{FC}\right) \geqslant 1$, according to the method described by Audic and Claverie [46].

\subsection{The Phylogenetic Relationship of Sequence}

Another 6 genes from 2 species of Oryza genus, including Oryza sativa Japonica Group (GenBank: BAD31281, BAD01425, NP_001061476) and Oryza sativa Indica Group (GenBank: Np_001067785, BAI39872, BAI39873), were downloaded from NCBI [47]. Sequence alignments were performed using the CLUSTAL W program set in MEGA 5.0 program (Tamura, Tokyo, Japan). Phylogenetic analyses and statistical neighbour-joining bootstrap tests of the phylogenies in which the Bootstrap number is 200 were carried out using the MEGA package.

\section{Conclusions}

The present study used de novo transcriptome analysis to determine the disease-resistance genes in the three tissues (i.e., leaves, stems and roots) of O. officinalis, an important wild species in the Oryza genus with high resistance to many diseases. Through de novo assembly, 137,229 contigs were obtained ranging from 200 to 19,214 bp with an N50 of 2331 bp using Illumina HiSeq 2000 platform. A total of 88,249 contigs were annotated with gene descriptions by sequence similarity searching against a non-redundant protein database, and 75,589 transcripts were further assigned to GO terms. Based on the KEGG pathway assignments, we identified 22 contigs involved in plant-pathogen interaction, which includes PAMP-triggered immunity and effector-triggered immunity. The results from expression analysis of genes related to plant-pathogen interaction in different tissues showed that the majority of genes were all expressed in the three tissues. Candidate genes for plant hormone regulation pathways were also identified. In addition, two kinds of rice bacterial blight-resistant genes in O. officinalis, including two Xa1 genes and three Xa26 genes, were also identified. Both Xa1 genes showed the highest expression level in the stem, whereas one Xa26 gene was expressed dominantly in leaves and another Xa26 genes displayed low expression level in all three tissues. These transcriptome data have led to the identification of a number of candidate genes and functional elements determining plant-pathogen interaction and plant hormone regulation pathways associated with disease resistance, which will accelerate functional genomic studies of O. officinalis and genetic improvement efforts for cultivated rice in the future.

Supplementary Materials: Supplementary Materials are available online at www.mdpi.com/1422-0067/ 16/12/26178/s1.

Acknowledgments: This work is supported by a program of the National Natural Science Foundation of China-Yunnan combination (U1302265) and a key project of Yunnan (2012HA002).

Author Contributions: Yizheng Zhang, Bin He, Zaiquan Cheng conceived and designed the project. Bin He, Xiang Tao and Yinghong Gu performed the experiments. Bin He, Changhe Wei, Jian Fu and Xiaojie Cheng analyzed the data. Bin He and Yizheng Zhang wrote the paper. All authors read and approved the final manuscript.

Conflicts of Interest: The authors declare no conflict of interest.

Availability of Supporting Data: The full data sets of O. officinalis leaves, stems and roots have been submitted to NCBI SRA databases under Accession SRR2102055, SRR2102056 and SRR2102057.

\section{References}

1. Fu, X.L.; Lu, Y.G.; Liu, X.D.; Li, J.Q.; Zhao, X.J. Cytological behavior of hybridization barriers between Oryza sativa and Oryza officinalis. Agric. Sci. China 2011, 10, 1489-1500.

2. Zhang, W.; Dong, Y.; Yang, L.; Ma, B.; Ma, R.; Huang, F.; Wang, C.; Hu, H.; Li, C.; Yan, C.; et al. Small brown planthopper resistance loci in wild rice (Oryza officinalis). Mol. Genet. Genom. 2014, 289, 373-382. [CrossRef] [PubMed]

3. Devanna, N.B.; Vijayan, J.; Sharma, T.R. The blast resistance gene Pi54of cloned from Oryza officinalis interacts with Avr-Pi54 through its novel non-LRR domains. PLoS ONE 2014. [CrossRef] [PubMed] 
4. Chen, J.; Huang, D.R.; Wang, L.; Liu, G.J.; Zhuang, J.Y. Identification of quantitative trait loci for resistance to whitebacked planthopper, Sogatella furcifera, from an interspecific cross Oryza sativa $\times$ O. rufipogon. Breed. Sci. 2010, 60, 153-159. [CrossRef]

5. Tan, G.X.; Ren, X.; Weng, Q.-M.; Shi, Z.-Y.; Zhu, L.-L.; He, G.-C. Mapping of a new resistance gene to bacterial blight in rice line introgressed from Oryza officinalis. Acta Genet. Sin. 2004, 31, 724-729. [PubMed]

6. Jacquemin, J.; Bhatia, D.; Singh, K.; Wing, R.A. The international Oryza map alignent project: Development of a genus-wide comparative genomics platform to help solve the 9 billion-people question. Curr. Opin. Plant Biol. 2013, 16, 147-156. [CrossRef] [PubMed]

7. Bao, Y.; Xu, S.; Jing, X.; Meng, L.; Qin, Z. De novo assembly and characterization of Oryza officinalis leaf transcriptome by using RNA-seq. BioMed Res. Int. 2015. [CrossRef] [PubMed]

8. Jeung, J.; Kim, B.; Cho, Y.; Han, S.; Moon, H.; Lee, Y.; Jena, K. A novel gene, Pi40 ( $t$ ), linked to the DNA markers derived from NBS-LRR motifs confers broad spectrum of blast resistance in rice. Theor. Appl. Genet. 2007, 115, 1163-1177. [CrossRef] [PubMed]

9. Sanchez, P.L.; Wing, R.A.; Brar, D.S. The wild relative of rice: genomes and genomics. In Genetics and Genomics of Rice; Springer: New York, NY, USA, 2013; pp. 9-25.

10. Huang, Z.; He, G.; Shu, L.; Li, X.; Zhang, Q. Identification and mapping of two brown planthopper resistance genes in rice. Theor. Appl. Genet. 2001, 102, 929-934. [CrossRef]

11. Boller, T.; He, S.Y. Innate immunity in plants: an arms race between pattern recognition receptors in plants and effectors in microbial pathogens. Science 2009, 324, 742. [CrossRef] [PubMed]

12. Jones, J.D.; Dangl, J.L. The plant immune system. Nature 2006, 444, 323-329. [CrossRef] [PubMed]

13. Zhang, J.; Liu, W.; Han, H.; Song, L.; Bai, L.; Gao, Z.; Zhang, Y.; Yang, X.; Li, X.; Gao, A.; et al. De novo transcriptome sequencing of Agropyron cristatum to identify available gene resources for the enhancement of wheat. Genomics 2015, 106, 129-136. [CrossRef] [PubMed]

14. Goff, S.A.; Ricke, D.; Lan, T.-H.; Presting, G.; Wang, R.; Dunn, M.; Glazebrook, J.; Sessions, A.; Oeller, P.; Varma, H.; et al. A draft sequence of the rice genome (Oryza sativa L. ssp. japonica). Science 2002, 296, 92-100. [CrossRef] [PubMed]

15. Project IRGS. The map-based sequence of the rice genome. Nature 2005, 436, 793-800.

16. Cho, S.; Ok, S.; Jeung, J.; Shim, K.; Jung, K.; You, M.; Kang, K.; Chung, Y.; Choi, H.; Moon, H.; et al. Comparative analysis of 5,211 leaf ESTs of wild rice (Oryza minuta). Plant Cell Rep. 2004, 22, 839-847. [CrossRef] [PubMed]

17. Lu, T.; Yu, S.; Fan, D.; Mu, J.; Shangguan, Y.; Wang, Z.; Minobe, Y.; Lin, Z.; Han, B. Collection and comparative analysis of 1888 full-length cDNAs from wild rice Oryza rufipogon Griff. W1943. DNA Res. 2008, 15, 285-295. [CrossRef] [PubMed]

18. Yang, H.; Hu, L.; Hurek, T.; Reinhold-Hurek, B. Global characterization of the root transcriptome of a wild species of rice, Oryza longistaminata, by deep sequencing. BMC Genom. 2010, 11, 705. [CrossRef] [PubMed]

19. He, B.; Zhao, S.; Chen, Y.; Cao, Q.; Wei, C.; Cheng, X.; Zhang, Y. Optimal assembly strategies of transcriptome related to ploidies of eukaryotic organisms. BMC Genom. 2015, 16, 65. [CrossRef] [PubMed]

20. Zhang, H.-B.; Xia, E.-H.; Huang, H.; Jiang, J.-J.; Liu, B.-Y.; Gao, L.-Z. De novo transcriptome assembly of the wild relative of tea tree (Camellia taliensis) and comparative analysis with tea transcriptome identified putative genes associated with tea quality and stress response. BMC Genom. 2015, 16, 298. [CrossRef] [PubMed]

21. Choi, H.W.; Hwang, B.K. Molecular and cellular control of cell death and defense signaling in pepper. Planta 2015, 241, 1-27. [CrossRef] [PubMed]

22. Zipfel, C.; Robatzek, S.; Navarro, L.; Oakeley, E.J.; Jones, J.D.; Felix, G.; Boller, T. Bacterial disease resistance in Arabidopsis through flagellin perception. Nature 2004, 428, 764-767. [CrossRef] [PubMed]

23. Ishikawa, K.; Yamaguchi, K.; Sakamoto, K.; Yoshimura, S.; Inoue, K.; Tsuge, S.; Kojima, C.; Kawasaki, T. Bacterial effector modulation of host E3 ligase activity suppresses PAMP-triggered immunity in rice. Nat. Commun. 2014, 5, 5430. [CrossRef] [PubMed]

24. Yamaguchi, K.; Yamada, K.; Ishikawa, K.; Yoshimura, S.; Hayashi, N.; Uchihashi, K.; Ishihama, N.; Kishi-Kaboshi, M.; Takahashi, A.; Tsuge, S.; et al. A receptor-like cytoplasmic kinase targeted by a plant pathogen effector is directly phosphorylated by the chitin receptor and mediates rice immunity. Cell Host Microbe 2013, 13, 347-357. [CrossRef] [PubMed] 
25. Liu, B.; Li, J.-F.; Ao, Y.; Qu, J.; Li, Z.; Su, J.; Zhang, Y.; Liu, J.; Feng, D.; Qi, K.; et al. Lysin motif-containing proteins LYP4 and LYP6 play dual roles in peptidoglycan and chitin perception in rice innate immunity. Plant Cell 2012, 24, 3406-3419. [CrossRef] [PubMed]

26. Katsir, L.; Chung, H.S.; Koo, A.J.; Howe, G.A. Jasmonate signaling: a conserved mechanism of hormone sensing. Curr. Opin. Plant Biol. 2008, 11, 428-435. [CrossRef] [PubMed]

27. Lugtenberg, B.J.; Chin-A-Woeng, T.F.; Bloemberg, G.V. Microbe-plant interactions: Principles and mechanisms. Antoni. Leeuwe. 2002, 81, 373-383. [CrossRef]

28. Katou, S.; Asakura, N.; Kojima, T.; Mitsuhara, I.; Seo, S. Transcriptome analysis of WIPK/SIPK-suppressed plants reveals induction by wounding of disease resistance-related genes prior to the accumulation of salicylic acid. Plant Cell Physiol. 2013, 54, 1005-1015. [CrossRef] [PubMed]

29. Loake, G.; Grant, M. Salicylic acid in plant defence-The players and protagonists. Curr. Opin. Plant Biol. 2007, 10, 466-472. [CrossRef] [PubMed]

30. Tang, J.; Wang, F.; Wang, Z.; Huang, Z.; Xiong, A.; Hou, X. Characterization and co-expression analysis of WRKY orthologs involved in responses to multiple abiotic stresses in Pak-choi (Brassica campestris ssp. chinensis). BMC Plant Biol. 2013, 13, 188. [CrossRef] [PubMed]

31. Tao, Z.; Liu, H.; Qiu, D.; Zhou, Y.; Li, X.; Xu, C.; Wang, S. A pair of allelic WRKY genes play opposite roles in rice-bacteria interactions. Plant Physiol. 2009, 151, 936-948. [CrossRef] [PubMed]

32. Mauch-Mani, B.; Mauch, F. The role of abscisic acid in plant-pathogen interactions. Curr. Opin. Plant Biol. 2005, 8, 409-414. [CrossRef] [PubMed]

33. Umezawa, T.; Sugiyama, N.; Mizoguchi, M.; Hayashi, S.; Myouga, F.; Yamaguchi-Shinozaki, K.; Ishihama, Y.; Hirayama, T.; Shinozaki, K. Type 2C protein phosphatases directly regulate abscisic acid-activated protein kinases in Arabidopsis. Proc. Natl. Acad. Sci. USA 2009, 106, 17588-17593. [CrossRef] [PubMed]

34. Sundaram, R.M.; Chatterjee, S.; Oliva, R.; Laha, G.S.; Cruz, C.V.; Leach, J.E.; Sonti, R.V. Update on Bacterial Blight of Rice: Fourth. Rice 2014, 7, 12. [CrossRef] [PubMed]

35. Tao, X.; Gu, Y.-H.; Wang, H.-Y.; Zheng, W.; Li, X.; Zhao, C.W.; Zhang, Y.Z. Digital gene expression analysis based on integrated de novo transcriptome assembly of sweet potato [Ipomoea batatas (L.) Lam.]. PLoS ONE 2012. [CrossRef] [PubMed]

36. Davis, M.P.; van Dongen, S.; Abreu-Goodger, C.; Bartonicek, N.; Enright, A.J. Kraken: A set of tools for quality control and analysis of high-throughput sequence data. Methods 2013, 63, 41-49. [CrossRef] [PubMed]

37. FastQC: A Quality Control Tool for High Throughput Sequence Data. Available online: http://www.bioinformatics.babraham.ac.uk/projects/fastqc/ (accessed on 1 November 2015).

38. Haas, B.J.; Papanicolaou, A.; Yassour, M.; Grabherr, M.; Blood, P.D.; Bowden, J.; Couger, M.B.; Eccles, D.; Li, B.; Lieber, M.; et al. De novo transcript sequence reconstruction from RNA-seq using the Trinity platform for reference generation and analysis. Nat. Protoc. 2013, 8, 1494-1512. [CrossRef] [PubMed]

39. Langmead, B.; Salzberg, S.L. Fast gapped-read alignment with Bowtie 2. Nat. Method 2012, 9, 357-359. [CrossRef] [PubMed]

40. Zhang, J.; Ruhlman, T.A.; Mower, J.P.; Jansen, R.K. Comparative analyses of two Geraniaceae transcriptomes using next-generation sequencing. BMC Plant Biol. 2013, 13, 228. [CrossRef] [PubMed]

41. Conesa, A.; Götz, S.; García-Gómez, J.M.; Terol, J.; Talón, M.; Robles, M. Blast2GO: A universal tool for annotation, visualization and analysis in functional genomics research. Bioinformatics 2005, 21, 3674-3676. [CrossRef] [PubMed]

42. WEGO. Available online: http://wego.genomics.org.cn/cgi-bin/wego/index.pl (accessed on 1 November 2015).

43. Kanehisa, M.; Goto, S. KEGG: Kyoto encyclopedia of genes and genomes. Nucleic Acids Res. 2000, 28 , $27-30$. [CrossRef] [PubMed]

44. KAAS. Available online: http://www.genome.jp/kegg/kaas/ (accessed on 1 November 2015).

45. Bateman, A.; Coin, L.; Durbin, R.; Finn, R.D.; Hollich, V.; Griffiths-Jones, S.; Khanna, A.; Marshall, M.; Moxon, S.; Sonnhammer, E.; et al. The Pfam protein families database. Nucleic Acids Res. 2004, 32, D138-D141. [CrossRef] [PubMed] 
46. Audic, S.; Claverie, J.-M. The significance of digital gene expression profiles. Genom. Res. 1997, 7, 986-995.

47. NCBI. Available online: http://www.ncbi.nlm.nih.gov (accessed on 1 November 2015).

(C) 2015 by the authors; licensee MDPI, Basel, Switzerland. This article is an open access article distributed under the terms and conditions of the Creative Commons by Attribution (CC-BY) license (http://creativecommons.org/licenses/by/4.0/). 